\title{
Telescoping Solar Array Concept for Achieving High Packaging Efficiency
}

\author{
Martin Mikulas ${ }^{1}$ \\ National Institute of Aerospace, Hampton, VA 23666 \\ and \\ Richard Pappa², Jay Warren ${ }^{3}$, and Geoff Rose ${ }^{3}$ \\ NASA Langley Research Center, Hampton, VA 23681
}

\begin{abstract}
Lightweight, high-efficiency solar arrays are required for future deep space missions using high-power Solar Electric Propulsion (SEP). Structural performance metrics for state-of-theart 30-50 kW flexible blanket arrays recently demonstrated in ground tests are approximately $40 \mathrm{~kW} / \mathrm{m}^{3}$ packaging efficiency, $150 \mathrm{~W} / \mathrm{kg}$ specific power, $0.1 \mathrm{~Hz}$ deployed stiffness, and $0.2 \mathrm{~g}$ deployed strength. Much larger arrays with up to a megawatt or more of power and improved packaging and specific power are of interest to mission planners for minimizing launch and life cycle costs of Mars exploration. A new concept referred to as the Compact Telescoping Array (CTA) with $60 \mathrm{~kW} / \mathrm{m}^{3}$ packaging efficiency at $1 \mathrm{MW}$ of power is described herein. Performance metrics as a function of array size and corresponding power level are derived analytically and validated by finite element analysis. Feasible CTA packaging and deployment approaches are also described. The CTA was developed, in part, to serve as a NASA reference solar array concept against which other proposed designs of $50-1000 \mathrm{~kW}$ arrays for future high-power SEP missions could be compared.
\end{abstract}

\section{Nomenclature}

a

a1

a2

$\mathrm{b}$

B

$=$ Ratio of array acceleration to g

$=$ Width of lateral end beam

$=$ Width of Z-folded blanket $=\mathrm{tpb}$

$=$ Width of array primary support box beam

$=$ Width of array wing

= Blanket packaging factor

$=$ Tip deflection of array wing subject to an acceleration, a

$=$ Modulus of array primary support box beam

$=$ Factor defining aspect ratio of array wing, L/B

= Array wing cantilever frequency

$=$ Gravitational constant

$=$ Height of array primary support box beam

$=$ Thickness of honeycomb core

$=$ Moment of inertia of array primary support box beam

$=$ Length of array blanket

$=$ Factor to account for additional package length due to telescoping structure

$=$ Mass per unit length of the array

$=$ Total mass of an array wing blanket

\footnotetext{
${ }^{1}$ Senior Research Fellow, AIAA Fellow.

${ }^{2}$ Research Engineer, Structural Dynamics Branch, Mail Stop 230, AIAA Associate Fellow.

${ }^{3}$ Research Engineer, Structural Dynamics Branch, Mail Stop 230.
} 


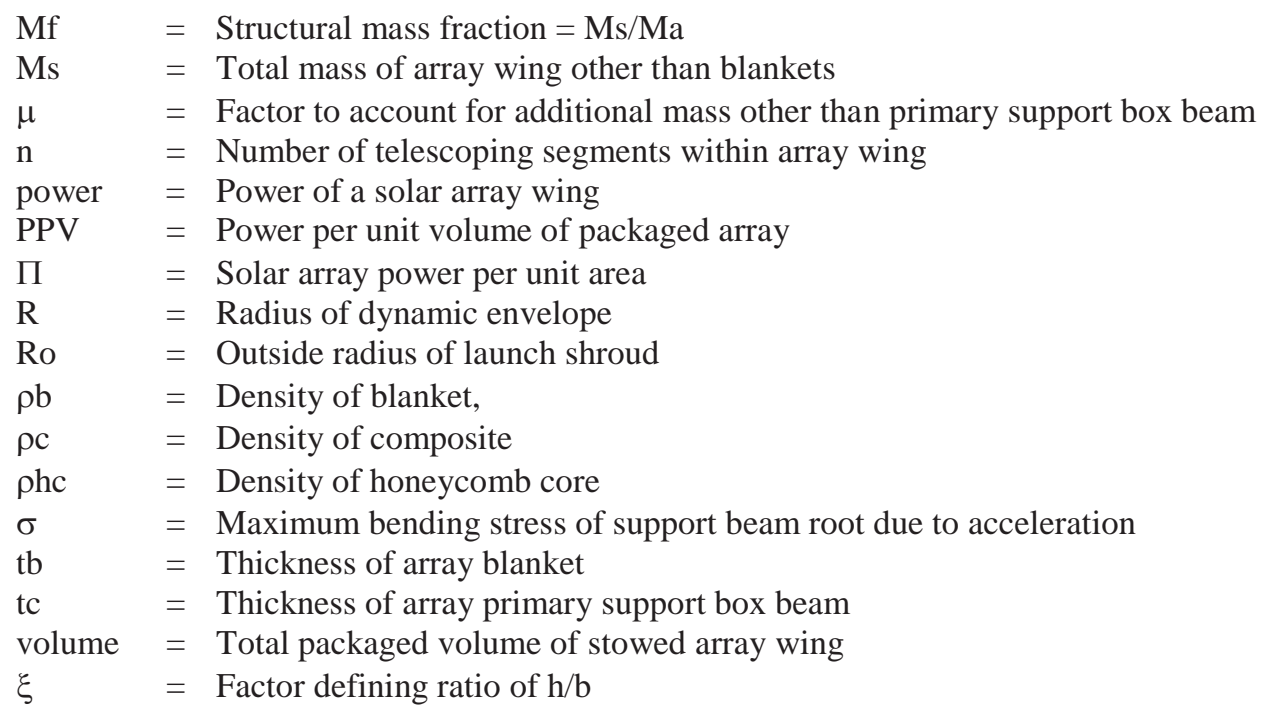

\section{Introduction}

Solar Electric Propulsion (SEP) provides a fuel-efficient means of in-space propulsion and is widely accepted as a leading near-term solution for exploration missions to asteroids and elsewhere..$^{1-3}$ The primary disadvantage of using SEP instead of traditional chemical systems for in-space propulsion is its low thrust and correspondingly long trip times especially for hauling massive cargo. However, because electric propulsion thrust is directly proportional to the supplied electrical power, the thrust can be increased using larger solar arrays.

Near-term SEP missions such as the Asteroid Redirect Mission (ARM) will use solar arrays in the 30-50 kW power class, which requires about 100 to $170 \mathrm{~m}^{2}$ of deployed surface area (50 to $85 \mathrm{~m}^{2}$ per wing) assuming $300 \mathrm{~W} / \mathrm{m}^{2}$ power production at the beginning of life (BOL) at 1 astronomical unit (AU). These sizes can be achieved with existing technology as demonstrated by the successfully developed ATK MegaFlex and DSS ROSA/Mega-ROSA engineering development units under recent NASA contracts. ${ }^{4}$ One important caveat related to the confidence with which these new arrays can be flown in space, however, is that they use fundamentally different structures technology than most existing spacecraft solar arrays. Specifically, the new ATK and DSS arrays use flexible substrates (tensioned blankets) rather than better-understood or more traditional rigid-panel substrates. Flexible-substrate designs package more compactly and are expected to be required for power levels exceeding about $25 \mathrm{~kW}$ because of launch fairing size limitations. One well-known example of a large solar array using tensioned flexible substrates is the International Space Station (ISS) solar arrays. Although some problems were encountered with the ISS arrays during some deployments and retractions ${ }^{5}$, they do represent a heritage design for large tensioned solar arrays. In the present paper, perturbations to the flight-proven ISS solar arrays are made to achieve a desired high solar array packaging efficiency of $60 \mathrm{~kW} / \mathrm{m}^{3}$ at BOL for proposed deep-space missions requiring electrical power from $30 \mathrm{~kW}$ up to $1 \mathrm{MW}$ or more.

A fundamental question that arises is how large can solar arrays for future SEP-powered vehicles be, considering the practicalities of finite launch vehicle fairing sizes and payload mass limits while ensuring reliable deployment and operation of expansive, lightweight solar array structures in space? Key design characteristics including launch mass and volume as well as deployed structural properties (strength, stiffness, etc.) need to be well defined at these unusually large sizes to enable rational mission system analyses. Solar arrays capable of producing many hundreds or thousands of kilowatts of electrical power have been recently proposed by mission planners for hauling cargo and possibly crew to Mars and elsewhere. ${ }^{6}$

In this study a novel approach is taken for the design of large flexible-substrate solar arrays. The power per unit volume (PPV) of the solar array is constrained to a desired level, after which achievable values of stiffness (fundamental natural frequency) and strength (acceleration load capability) are determined for practical values of structural mass fraction, Mf. This approach is motivated by recent mission studies indicating that ultra-compact

American Institute of Aeronautics and Astronautics 
packaging can be an enabling requirement for launch of huge, megawatt-class arrays, while requirements for array structural frequency and acceleration capability may vary with mission design. Also, there exist alternate technologies that could be incorporated to deal with frequency and acceleration requirements for large arrays. These technologies include but are not limited to the use of micro-thrusters and other active control strategies and guy support cables. In the present paper, the mass fractions for a reference solar array are presented as a function of, structural frequency, packaging efficiency, and power level.

\section{Solar Array Definitions and Assumptions}

The baseline power-per-unit-volume, PPV, goal chosen in this study is $60 \mathrm{~kW} / \mathrm{m}^{3}$. This goal is about $50 \%$ higher than achievable by state-of-the-art solar array designs at the largest existing sizes of 30-50 kW. Furthermore, solar array packaging efficiency normally decreases with increasing solar array size, making the $60 \mathrm{~kW} / \mathrm{m}^{3}$ goal even harder for sizes up to $1 \mathrm{MW}$. To achieve the $60 \mathrm{~kW} / \mathrm{m}^{3}$ packaging goal, major improvements will be required of existing concepts or new design concepts and approaches will be required.

The major solar array definitions and assumptions used in the current study are shown in Fig. 1. Using assumed values for BOL power-per-unit-area (П) of $300 \mathrm{~W} / \mathrm{m}^{2}$ and a blanket thickness of $0.76 \mathrm{~mm}(0.030$ ") results in a blanket volume fraction of 0.15 . This means that for an assumed PPV of $60 \mathrm{~kW} / \mathrm{m}^{3}$, the array blanket will occupy $15 \%$ of the total stowed volume while the structure and all other parts of the solar array must fit into the remaining $85 \%$. The total volume $(\mathrm{V})$ of the packaged solar array is given by:

$$
\mathrm{V}=\text { Power } / \mathrm{PPV}
$$

For example, the volume occupied by a 1 MW array would be:

$$
\mathrm{V}=1,000,000 / 60,000=16.67 \mathrm{~m}^{3}
$$

Since the blanket occupies $15 \%$ of the volume, the remainder of the array, which includes all supporting structure, mechanisms, etc., must fit in a volume of $14.2 \mathrm{~m}^{3}$.

\section{Compact Telescoping Array (CTA)}

To achieve the desired high solar array packaging efficiency, it is essential to begin with a highly efficient structural form. In all structures that involve bending stiffness to achieve performance, the structural efficiency increases as the number of primary support members decreases. Therefore, the optimal structure has a single primary support member. The reason for this is that the most efficient approach for achieving bending stiffness is to make the structure deeper rather than distributing it over multiple members. ${ }^{7}$

The ISS solar array wing is such a concept. It has a single beam or column to provide the required out-of-plane bending stiffness and torsional stiffness and to provide the column capability to tension the arrays. The strengths and weaknesses of the ISS solar arrays are presented in Fig. 2. As is well known, all systems require design tradeoffs between the ideal optimum and a practical solution considering schedule, cost, and other factors. The ISS arrays were designed to be affordable for low earth orbit operations. Also with the ISS multiple flight assembly scenario using the Space Shuttle, packaging volume as well as structural mass were not critical. However, for deep-space missions such as Mars exploration, structural mass as well as packaging volume will be major design drivers. For such missions, advanced technologies such as ultra-lightweight composites and novel compactly packaged structures will provide major mission benefits.

The major strengths of the ISS solar array wing design are the extremely compact solar-cell-blanket packaging density of about $300 \mathrm{~kW} / \mathrm{m}^{3}$ (assuming the circa 1970 solar cells are replaced with modern $30 \%$ efficient cells) and the high structural efficiency achieved by using a single primary support member. However, the single-fold truss concept (that deploys in just one direction) selected for the primary support member and the relatively large volume

American Institute of Aeronautics and Astronautics 
of the blanket support arms result in an overall PPV of only $12 \mathrm{~kW} / \mathrm{m}^{3}$, again assuming $30 \%$ efficient cells. In the present study it was determined that the two changes to the ISS solar concept shown in Fig. 3 could result in major improvements in the PPV. These changes were to replace the single-fold deployable truss with a telescoping structure and to reduce the cross-section of the end support arms. Using a telescoping primary support structure integrates the function of the canister and the structure. The ISS canister is approximately $25 \%$ of the total solar array mass and is parasitic mass after the array deploys. The other major change required for achieving high packaging efficiency is development of a reduced volume support arm structure. This reduced volume cross-section member could be accomplished in numerous ways and will be the subject of further study.

A sketch of the proposed Compact Telescoping Array (CTA) is shown in Fig. 4. The telescoping primary support structure consists of an arbitrary number of segments (n) between the root and tip support arms with an additional segment at the root for a stand-off from the spacecraft and an additional segment at the tip to support guy cables as shown. For large power arrays, the end support arms will likely be sufficiently long that guy support cables will be needed to increase structural efficiency for carrying the blanket tension loads. Figure 4 also shows an end view of the packaged array. The blankets are z-folded between the root and the tip support arms. The resulting package is then folded to lie alongside the stowed telescoping primary support structure. The shaded region in the center of the telescoping column represents a linear actuator that would sequentially deploy the telescoping structure one segment at a time. The selection of a deployment device for the telescoping support structure will involve a tradeoff among numerous concepts and is beyond the scope of the present paper.

A photograph of the deploying ISS solar array blanket is shown on the left of Fig. 5. A sketch of the packaged cross-section of the blanket support arm is shown in the upper right of Fig. 5. The deep structural boxes located on each side of the z- folded cell blanket are required to withstand bending caused by the blanket tension loads. As mentioned previously, these boxes represent a large volume and are not compatible with the high packaging density sought in the present study. Thus, alternate approaches must be considered for providing adequate structure to withstand the blanket tension loads while being capable of compact packaging. The arm box structure could be collapsed as shown in the lower right of Fig. 5 or it could be a shear-collapsed truss. In the current study the blanket support arms are assumed to be supported by several tensioned cables, thus, enabling a smaller cross-section. Volumetric requirements for the support arm are included in the CTA design code presented in Appendix A.

The shaded region in the center of the telescoping column of Fig. 4 represents a linear-motor-type-device that deploys the telescoping structure. The selection of a deployment device for the telescoping support structure will involve a tradeoff among numerous concepts and is beyond the scope of the present paper. The deployment could either be sequential as suggested by ATK $^{8}$ in Fig. 6 or continuous as suggested by NG/ASTRO ${ }^{9}$ in Fig. 7.

A high level overview of the deployment sequence of the compact telescoping array is shown in Fig. 8. In the first sketch the central primary telescoping support structure is shown with the stowed solar arrays folded and lying along its sides to form a very compact package. In the second sketch a linear actuator is shown deploying a tip telescopic member that in turn pulls into place the stowed solar array blankets and blanket support arms. In the third sketch, after the blanket support arms reach their fully deployed state, the internal linear actuator continues to deploy subsequent telescoping sections as well as pulling out the Z-folded array blankets. This process is continued as shown in the fourth sketch until the Z-folded blankets are pulled straight and tensioned as needed to achieve the desired natural frequency. It is assumed that the array wing deployment process will be similar to that developed for the ISS.

\section{Compact Telescoping Array Sizing Approach and Results}

Representative sizes and shapes of the telescoping array concept considered in this design procedure are shown in Fig. 9. All arrays considered herein use a length-to-width wing aspect ratio of 4 to 1 . Further system studies will be required to identify the effects of aspect ratio for a given application. The wing sizes shown in Fig. 9 result from using a wing aspect ratio of 4 and a blanket power per unit area of $300 \mathrm{~W} / \mathrm{m}^{2}$ as indicated previously. The sketch of two ISS solar array wings as shown in Fig. 9 provide a size comparison.

American Institute of Aeronautics and Astronautics 
The Mathematica analysis and design code for the compact telescoping array is presented in Appendix A and will now be described. The approach taken for preliminary design of the compact telescoping solar array involves determining the packaged volume required to achieve the desired PPV, and then sizing the volume of all elements of the array to meet that goal. The number of segments chosen for the telescoping support structure is selected so the package length will accommodate the launch vehicle for the mission. This design procedure quantifies the volume of each of the individual components shown in Figs. 4, A-1, and A-2, and constrains the total volume to meet the required packaged volume.

The number of telescoping members is as shown in Fig. A-1. There are $\mathrm{n}$ telescoping segments within the dimensions, L, of the array blanket, and there is an additional segment at the root of the array that attaches to the spacecraft. The number of telescoping segments, n, was set equal to $(10 \mathrm{~L}) / 81$. The resulting total length of the solar array wing is $L(n+1)$. This value for $n$ was chosen to approximate various sizes of launch vehicles that may be used as a function of array size. Of course this value would have to be updated as more was understood about a specific mission application and launch vehicle size. For a given power level, a given array aspect ratio, $\eta$, and a given powerper-unit-area, $\Pi$, the length and width, $\mathrm{L}$ and $\mathrm{B}$, and the mass of the array, Ma, can be determined. As described above, this then determines the number of telescoping members, $n$. In the present study, it is assumed that the number of telescoping members, $\mathrm{n}$, is never less than 4 . For smaller arrays, this assumption would have to be investigated further.

The depth of the primary support beam is, h, while the width, b, is assumed to be $h / \xi$. As shown in Figs. A- 1 and A-2, the width of the blanket support arm, a1, is equal to $\mathrm{h} / \mathrm{C} 1$. The width of the Z-folded blanket package is simply the number of folds times the thickness of the blanket, tb, times a blanket packaging factor, BPF as given by the next to last equation in Appendix A2. The depths of both the blanket support arm and the Z-folded arrays are taken to be $\mathrm{h} / 2$ as indicated in Fig. A-2. By equating the sum of all of the array components to the total available volume, an equation for $\mathrm{h}$ can now be derived as shown in the fourth equation from the bottom of Appendix A2. After $\mathrm{h}$ is known, all other array dimensions can be determined.

Now that all of the array wing dimensions are known, the major remaining unknown is the thickness of the primary support beam. The beam thickness is determined by requiring the frequency of the cantilevered array wing to have a natural frequency, $\mathrm{f}$. Since the natural frequency is a function of the mass of the solar array blankets as well as the mass of the primary support structure beam, an iterative solution must be conducted to converge on the thickness of the beam. That procedure is shown in Appendix A3. After the thickness of the beam is determined, the structural mass as well as the structural mass fraction can be determined.

Two other checks are required to ensure that the resulting design is feasible. These checks involve the performance of the array subject to an acceleration, a. For a given acceleration the tip deflection, $\Delta$, must be reasonable and the box beam must not fail at the root.

\section{A. $500 \mathrm{~kW}$ wing example results}

An example set of results is presented for a $500 \mathrm{~kW}$ wing in Appendix A5 using the print statements in Appendix A4. For this example, the resulting structural mass fraction is 0.54 . The thickness required to achieve the prescribed frequency of $0.05 \mathrm{~Hz}$ is 0.041 inches and the associated beam depth is 30.9 inches. The beam root stress due to an acceleration, a, of 0.2 is $59.5 \mathrm{ksi}$ with an associated strain level of 0.0029 . Although this strain level is well within the capability of the M55J composite being used as an example, local buckling of the compression side of the box must be checked. A check of the compression side of the box at the root of the primary support beam showed that the composite thickness of 0.041 inches would result in wall buckling. In order to constrain buckling without increasing the wall thickness would require that the box beam wall be made of an open grid or sandwich construction. A buckling study was made of the beam assuming it was constructed with a sandwich wall. The results of the study showed that for this, worst case, situation (a large $500 \mathrm{~kW}$ wing with a 0.2 acceleration) that a sandwich with a 0.3 inch thick core would eliminate the buckling problem. This thickness of sandwich core only increased the structural mass fraction

American Institute of Aeronautics and Astronautics 
from 0.54 to 0.567 . Further checks of smaller arrays showed that the resulting mass penalty was even less. The alternate approach of eliminating wall buckling using open grid construction with the same average thickness could possibly result in a primary support beam with no local buckling mass penalty. Because of the relatively small penalty associated with wall buckling, it was no longer considered in the current study.

To provide some insight into the impact of the compact packaging associated with the $60 \mathrm{~kW} / \mathrm{m}^{3}$ goal of the current study, a single $500 \mathrm{~kW}$ solar array wing is shown inside of a 5 meter diameter launch shroud in Fig. 10. No attempt is made to show the array attached to an actual payload. However, the relatively small cross-section of the packaged solar array enables a wide variety of attachment options to be considered.

\section{B. Parametric Results}

To provide insight into major solar array design drivers, the solar array structural mass fraction is plotted as a function of frequency, f, power-per-unit-volume, PPV, and solar array wing power, in this section. Understanding these primary design drivers will assist in establishing rational design requirements for advanced missions requiring large solar arrays.

\section{Mass fraction versus frequency}

The structural mass fraction as a function of array natural frequency is presented in Fig. 11 for four selected power sizes while holding the power-per-unit-volume at a fixed value of $60 \mathrm{~kW} / \mathrm{m}^{3}$. An important finding illustrated in Fig. 11 is that the structural mass fraction (Mf) increases dramatically with increased frequency for the large solar arrays. In fact, for the $1 \mathrm{MW}$ solar array it is impossible to achieve a $0.1 \mathrm{~Hz}$ frequency for the assumed parameters of the study. This steep increase in structural mass fraction is primarily caused by the rather aggressive volumetric constraint of $60 \mathrm{~kW} / \mathrm{m}^{3}$. The volumetric constraint limits the depth of the primary support beam, thus, the bending stiffness required for obtaining a higher frequency can only be achieved in an inefficient fashion by increasing the beam wall thickness. On the other hand it should also be noted that for the much smaller $15 \mathrm{~kW}$ wing, a structural mass fraction of 0.5 or lower can be maintained out to a frequency of $0.3 \mathrm{~Hz}$. The lowest data point on each curve approximately represents a $0.2 \mathrm{~g}$ strength capability. In other words, the arrays cannot have a lower mass assuming they must also achieve a deployed strength of $0.2 \mathrm{~g}$.

\section{Mass fraction versus power-per-unit-volume, $P P V$}

In the previous section it was observed that a major driver for solar array structural mass fraction is the volumetric packaging factor. As the array is required to be stowed in a smaller volume, the resulting primary support structure is smaller and accordingly less efficient. The effect of the packaging factor, PPV, on the solar array structural mass fraction is shown in Figs. 12 and 13 for a $0.1 \mathrm{~Hz}$ and a $0.05 \mathrm{~Hz}$ required natural frequency. For a given wing power, the structural mass fraction decreases with increasing PPV until the minimum gage wall constraint is exceeded. After that occurs the structural mass fraction increases dramatically. The reason for this rapid increase in structural mass fraction is that the size of the main support beam is decreasing as depicted with the rectangular beam cross-sections shown for the $500 \mathrm{~kW}$ wing power case. As discussed before, the moment of inertia required to achieve the desired natural frequency can only be achieved by increasing the beam wall thickness. This is an inefficient way of achieving bending stiffness.

The dramatic change in structural mass fraction that results from reducing the required natural frequency from 0.1 $\mathrm{Hz}$ to $0.05 \mathrm{~Hz}$ is shown on Fig. 13. For this lower frequency, even the $500 \mathrm{~kW}$ solar array wing has a very reasonable structural mass fraction of 0.54 at the power-packaging goal of $60 \mathrm{~kW} / \mathrm{m}^{3}$.

The goal of the current study was to identify the feasibility of achieving large solar arrays with a power-packaging factor of $60 \mathrm{~kW} / \mathrm{m}^{3}$. For a reference, the structural mass fraction and packaging factor for the ISS $0.1 \mathrm{~Hz}$ natural frequency solar array wings is also shown in Figs. 12 and 13. Although the ISS wing structural mass fraction is reasonably good, the resulting power-packaging factor was only $12 \mathrm{~kW} / \mathrm{m}^{3}$. The ISS packaging factor is calculated assuming the solar array cells had an efficiency of $30 \%$.

American Institute of Aeronautics and Astronautics 


\section{Mass fraction versus solar array wing power level}

A confusing occurrence in solar array design is that in some cases the structural mass fraction has been observed to decrease with increasing power level. This situation can occur when the same thickness material or the same size mechanisms are used as the array size increases. This behavior is commonly referred to as minimum gage design effects. To study this effect and to provide insight into proper structural scaling for large solar arrays, the structural mass fraction for the compact telescoping array, (CTA), is plotted in Fig. 14 as a function of power level for three different values of required natural frequency, $0.1 \mathrm{~Hz}, 0.075 \mathrm{~Hz}$, and $0.05 \mathrm{~Hz}$. For all three of the frequency cases it can be seen that indeed the structural mass fraction does initially decrease as power levels increase. In the current design approach as shown in Appendix A, the only minimum gage quantity is the primary support structure wall thickness that is taken to be 0.02 inches. This simple model does, however, demonstrate the minimum gage trend that does exist for solar array wings with power levels less than $50 \mathrm{~kW}$ or so.

The major implication of this observation is that structural designs for solar arrays in the minimum gage region will not scale properly to larger designs. For the $0.1 \mathrm{~Hz}$, and the $0.075 \mathrm{~Hz}$ frequency cases, it can be seen that the structural mass fractions increase quite dramatically as soon as the minimum gage design constraint is exceeded. Again, the reason for this is that all designs are constrained to package within the volumetric constraint of $60 \mathrm{~kW} / \mathrm{m}^{3}$. This means that the primary support structure cannot get larger and that the only way to achieve the higher bending stiffness required is to increase the beam wall thickness. This is a mass inefficient way of achieving bending stiffness, thus, the rapid rise is structural mass fraction. From a structural point-of-view, the best way to eliminate the dramatic rise in structural mass fraction is to lower the required natural frequency. As can be seen for the $0.05 \mathrm{~Hz}$ frequency case, the structural mass fraction is rather flat over the wing power range out to $500 \mathrm{~kW}$.

The results of this study strongly emphasize the need to develop technologies that will enable the operation of large solar arrays in the natural frequency range of $0.05 \mathrm{~Hz}$. Examples of such technologies are micro thrusters, advanced active controls, and tension guy wires. Their consideration is beyond the scope of the current study.

\section{Finite Element Validation and Mode Shapes}

Since the dynamic analysis used in the preliminary design procedure is necessarily highly simplified, a parallel finite element analysis (FEA) of the arrays was conducted. Using results from the FEA, a first-order correction was applied to the analysis. The ABAQUS FEA model used is shown in Fig. 15. The adjustment made to the simple frequency equation in Appendix A was to apply a frequency adjustment factor, faf. Based on the FEA results, a value of 0.7 was chosen for faf.

A major feature of the model was that guy support cables were used to support the blanket support arms. It is believed that for large arrays, such cables will be required to keep cross-sectional dimensions of the blanket support arms to acceptable levels in order to maintain a high packaging efficiency. The inclusion of these guy cable increased the resulting structural mass fraction from the simple design procedure value of 0.54 to 0.57 as shown in Fig. 14 .

\section{Conclusions}

A novel structural concept has been developed to enable a very high packaging density for large solar arrays. The selected concept is a modified version of the flight-proven ISS solar arrays. The approach used to develop the new concept was to maintain the two design strengths of the ISS solar arrays while improving the two observed design weaknesses. Specifically, the Z-folded ISS solar array blankets package extremely well and the single primary support beam represents excellent structural form for mass efficiency. Two major improvements were incorporated to improve the solar array packaging efficiency. First, the single-fold-truss primary support beam was replaced with a compact telescoping structure. This has the result of integrating the canister with the structure, with a significant savings in both mass and volume. Second, it was found to be necessary to reduce the volume of the support arms that provides the blanket tension. These two changes improve the PPV performance of the solar array by a factor of 5 . Although the array shows promise for significantly improving packaging efficiency, additional design and development is required for the associated deployment mechanisms and to ensure the array meets other design requirements for launch loads,

American Institute of Aeronautics and Astronautics 
thermal environments, etc. Preliminary results of this study show that a $1 \mathrm{MW}$ deployable solar array with a PPV of $60 \mathrm{~kW} / \mathrm{m}^{3}$ is achievable with a structural mass fraction of 0.54 and fundamental natural frequency of $0.05 \mathrm{~Hz}$ using the newly developed array concept. The results of this study strongly emphasize the need to develop technologies that will enable the operation of large solar arrays in the natural frequency range of $0.05 \mathrm{~Hz}$. Examples of such technologies are micro thrusters, advanced active controls, and tension guy wires. Their consideration was beyond the scope of the current study.

\section{Acknowledgments}

This work was funded and inspired by Mr. Chuck Taylor, Principal Investigator for In-Space Propulsion and Space Power Generation in NASA's Space Technology Mission Directorate. The findings contribute to a megawatt solar array feasibility study funded by Mr. Taylor and the Game Changing Technology Development Program.

\section{References}

${ }^{1}$ Leone, D., "NASA Banking on Solar Electric Propulsion's Slow but Steady Push,” SpaceNews.com, May 20, 2013.

${ }^{2}$ Hoffman, D. J., et al., "Concept Design of High Power Solar Electric Propulsion Vehicles for Human Exploration," NASA/TM-2011-217281, Dec. 2011.

${ }^{3}$ Polk, J. E., et al., “An Overview of NASA's Electric Propulsion Programs, 2010-11,” Presented at the 32nd International Electric Propulsion Conference, Wiesbaden, Germany, Sept. 11-15, 2011.

${ }^{4}$ Advanced Solar Array Systems, http://www.nasa.gov/offices/oct/home/feature_sas.html, Oct. 31, 2012.

${ }^{5}$ Brady, T. K. and Sweeney, D. J., "International Space Station (ISS) Solar Array Wing (SAW) Lessons Learned, NASA TM2011-217064, Vols. 1 \& 2, March 2011.

${ }^{6}$ Raftery, M., et al., "An Affordable Mission to Mars," Presented at the 64th International Astronautical Congress, Beijing, China, Sept. 23-27, 2013.

${ }^{7}$ Kurland, R. and Stella, P., “Advanced Photovoltaic Solar Array Design and Performance,” Presented at the Aerospace Design Conference, Irvine, CA, Feb. 3-6, 1992.

${ }^{8}$ http://www.atk.com/products-services/telescoping-boom

${ }^{9}$ northropgrumman.com/BusinessVentures/AstroAerospace/Products/Pages/TelescopicBoom.aspx 


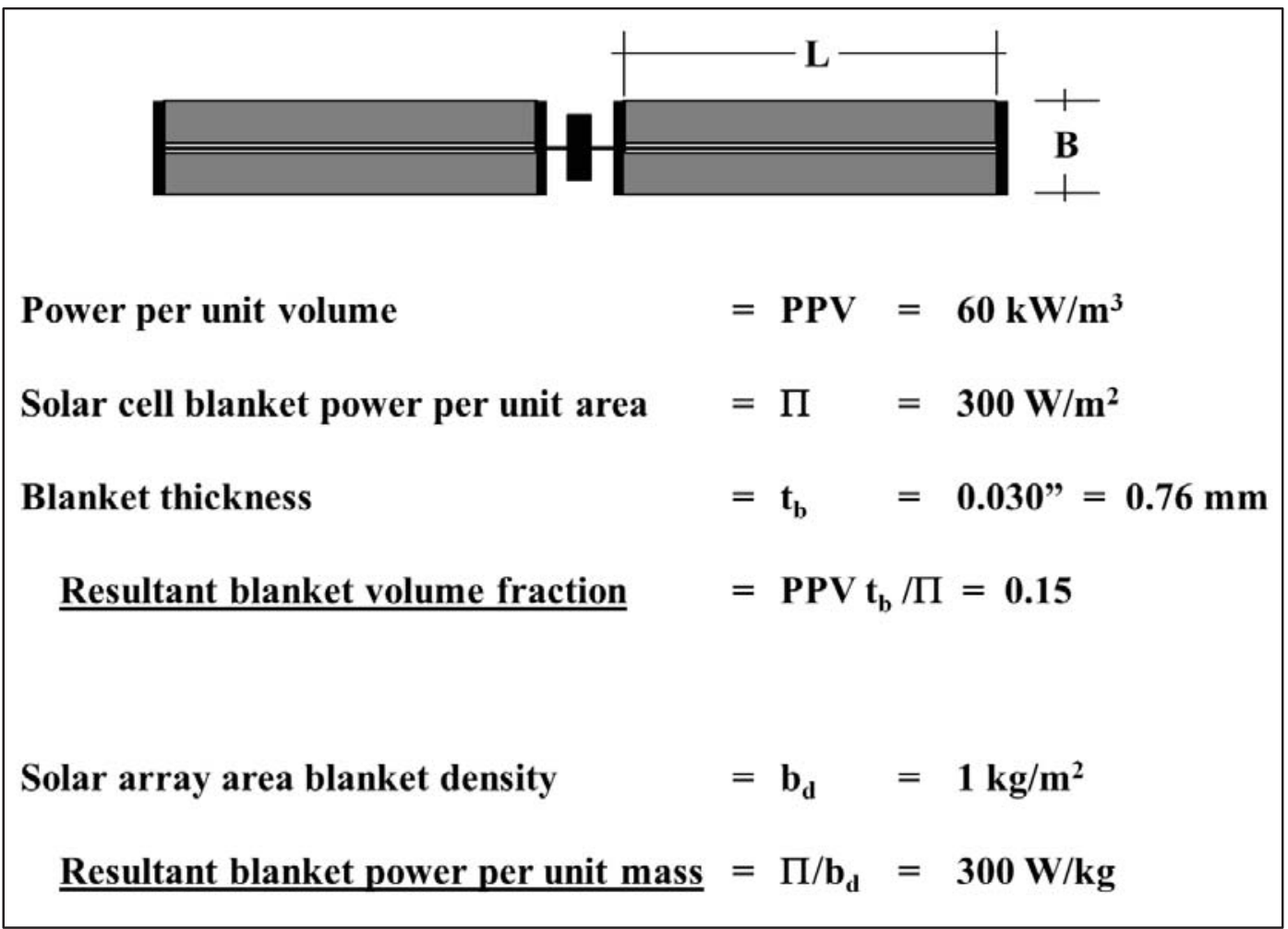

Figure 1. Solar array definitions and assumptions.

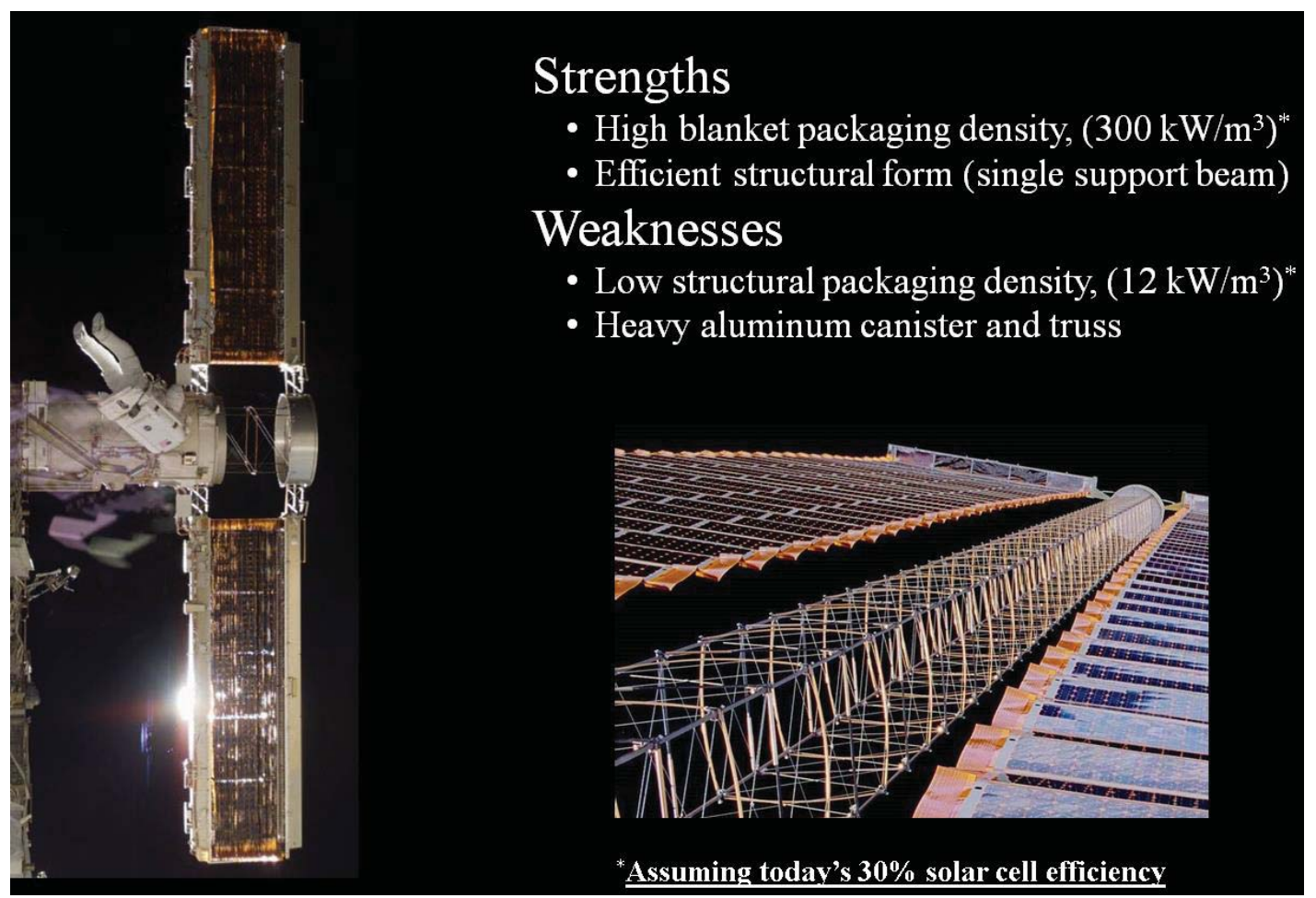

Figure 2. Characteristics of the international space station (ISS) solar arrays. 


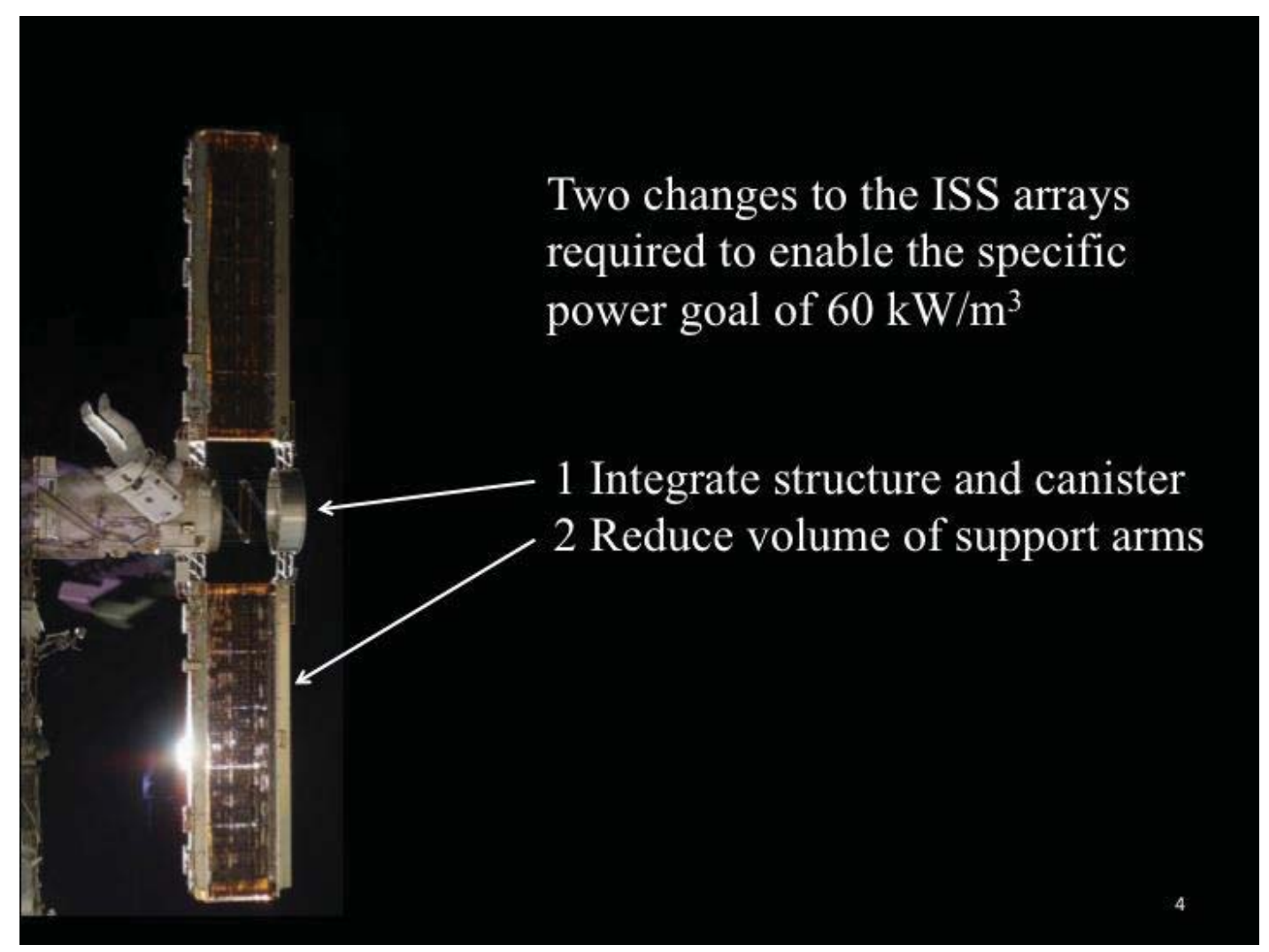

Figure 3. Approach used herein for improved solar array packaging.

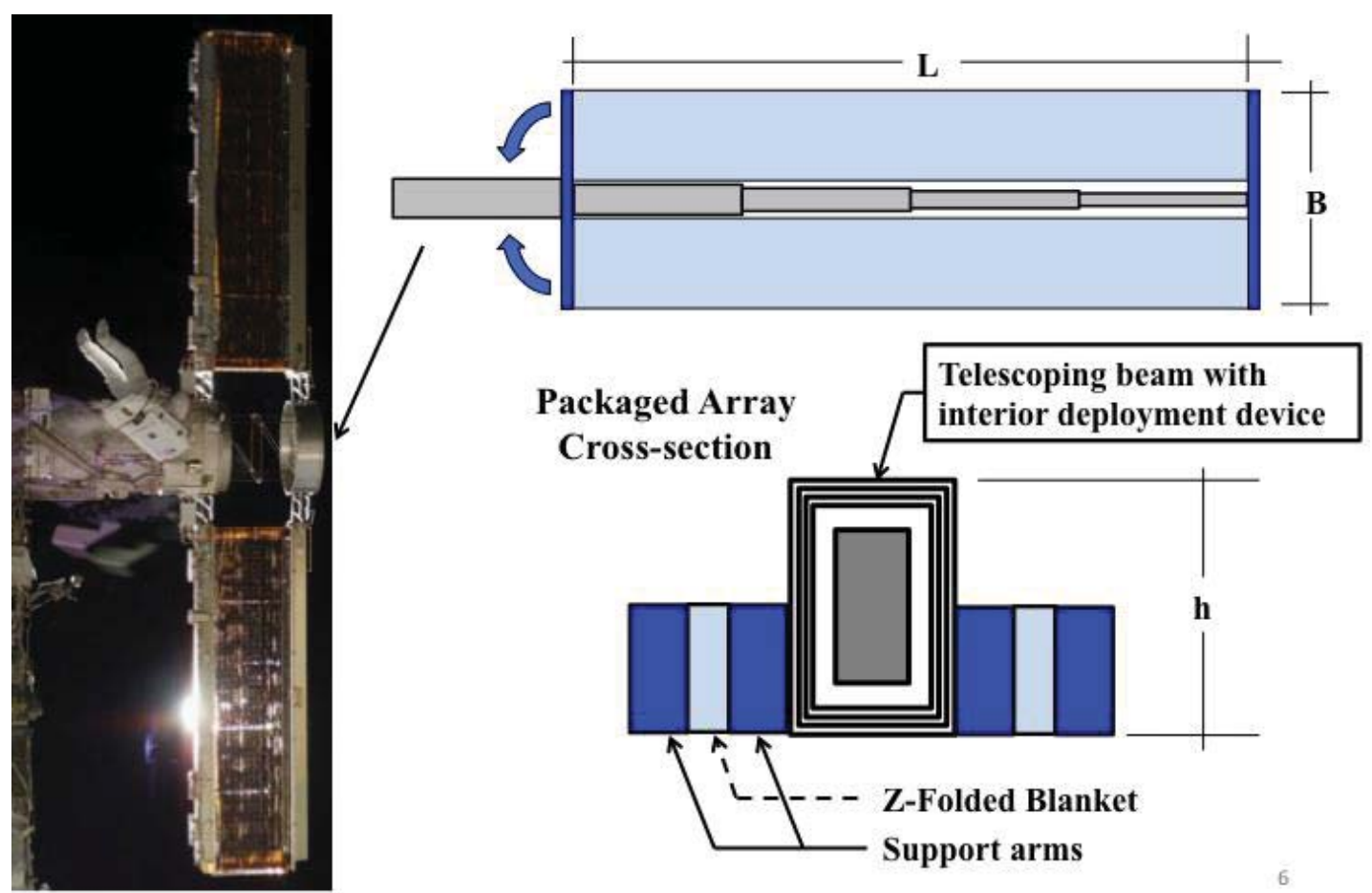

Figure 4. Compact telescoping array (CTA) concept. 

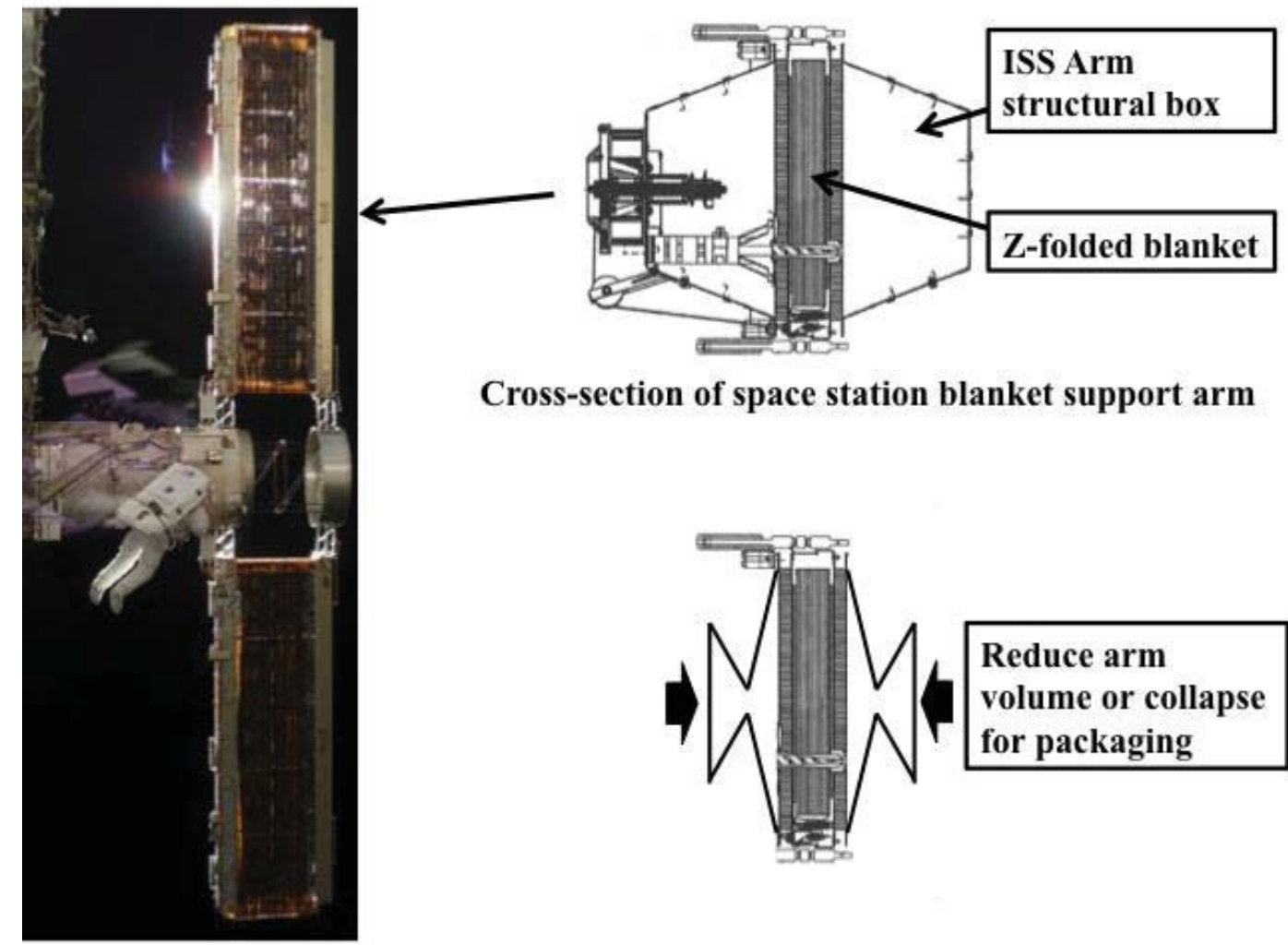

Cross-section of space station blanket support arm

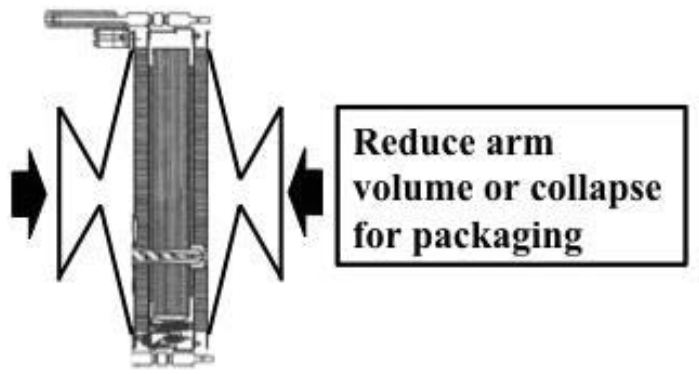

Figure 5. Photograph of deploying ISS solar array and cross-section of the ISS blanket support arms.

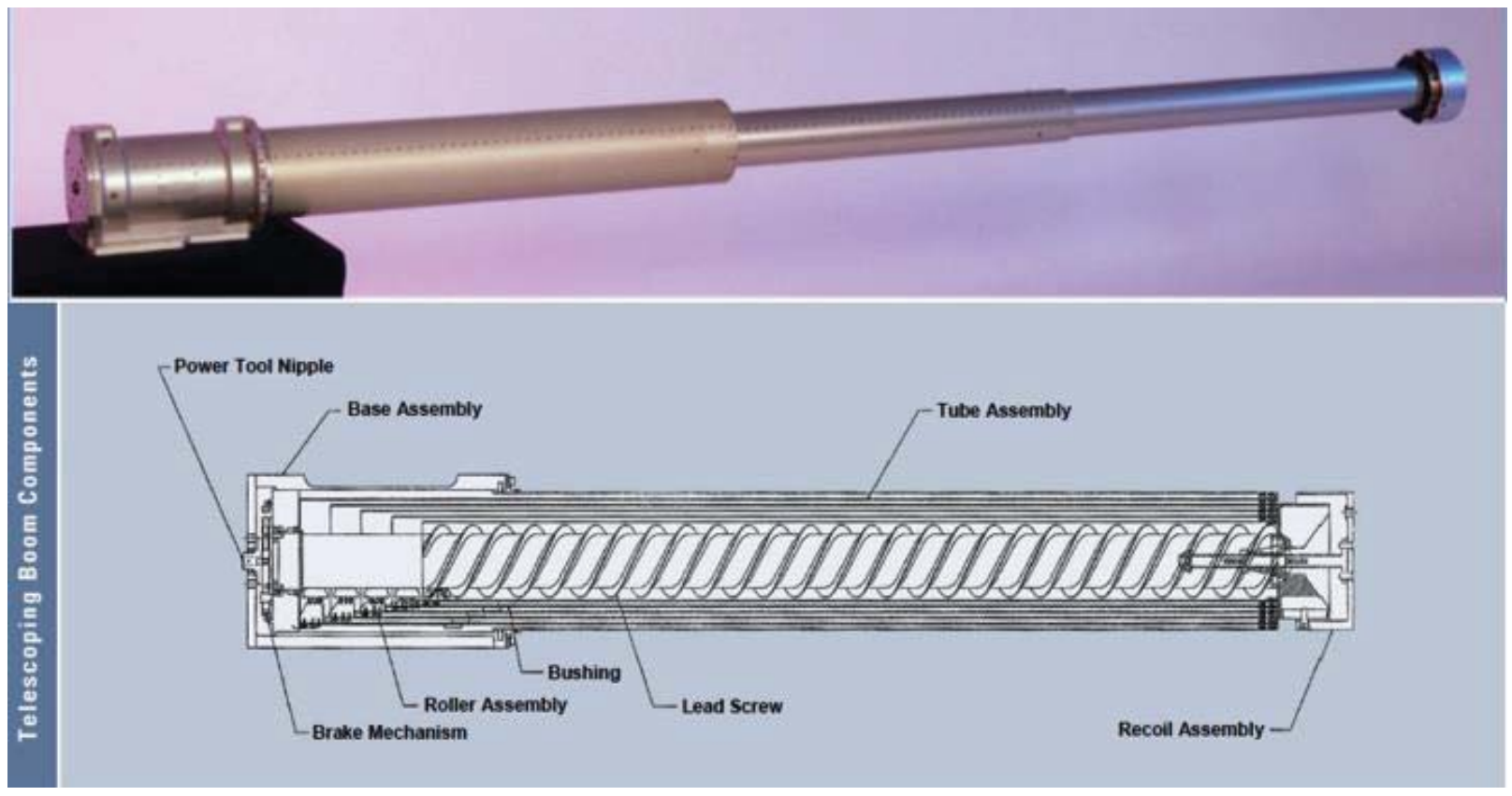

Figure 6. ATK telescoping structure. 

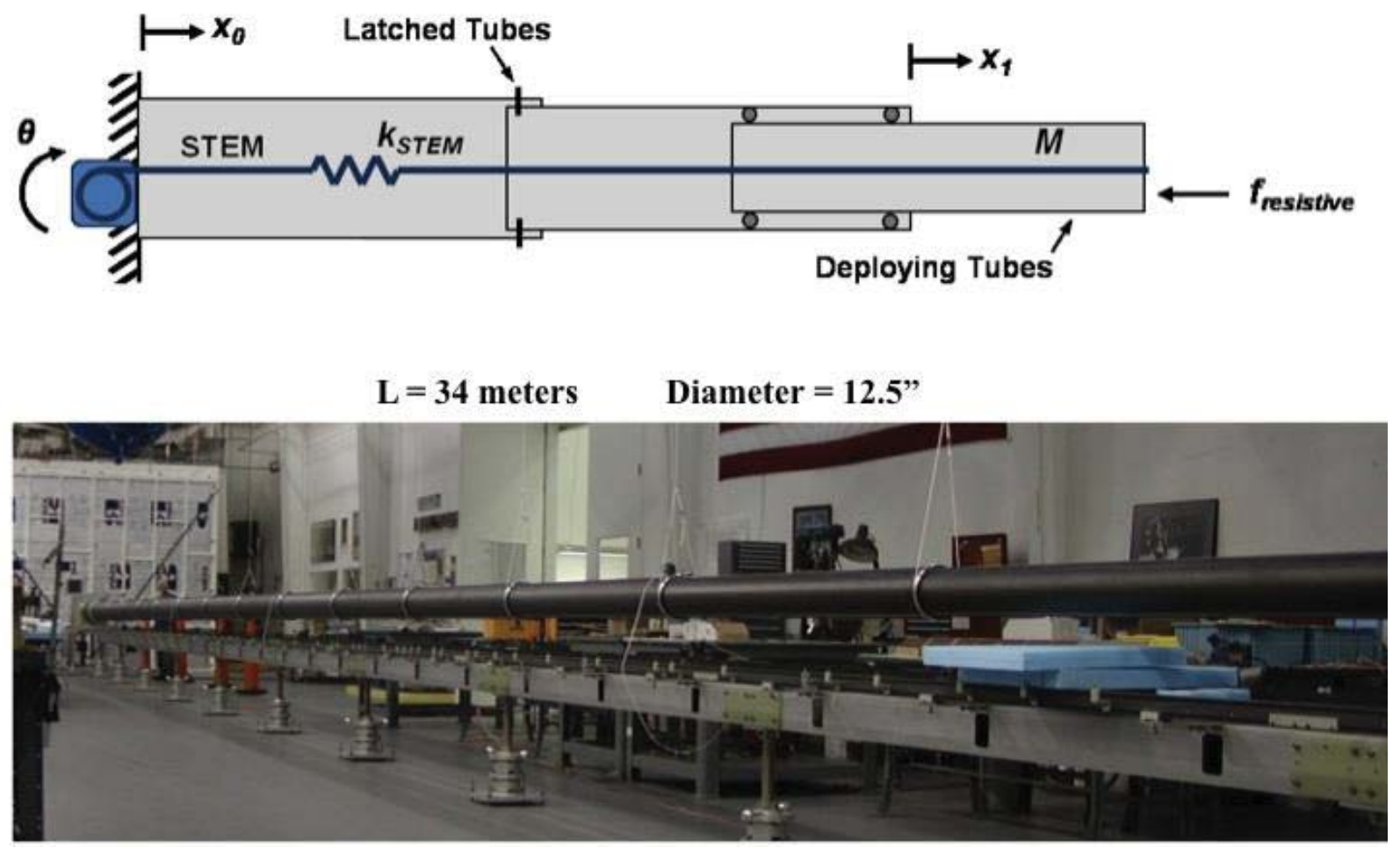

Figure 7. NG/ASTRO telescoping structure.
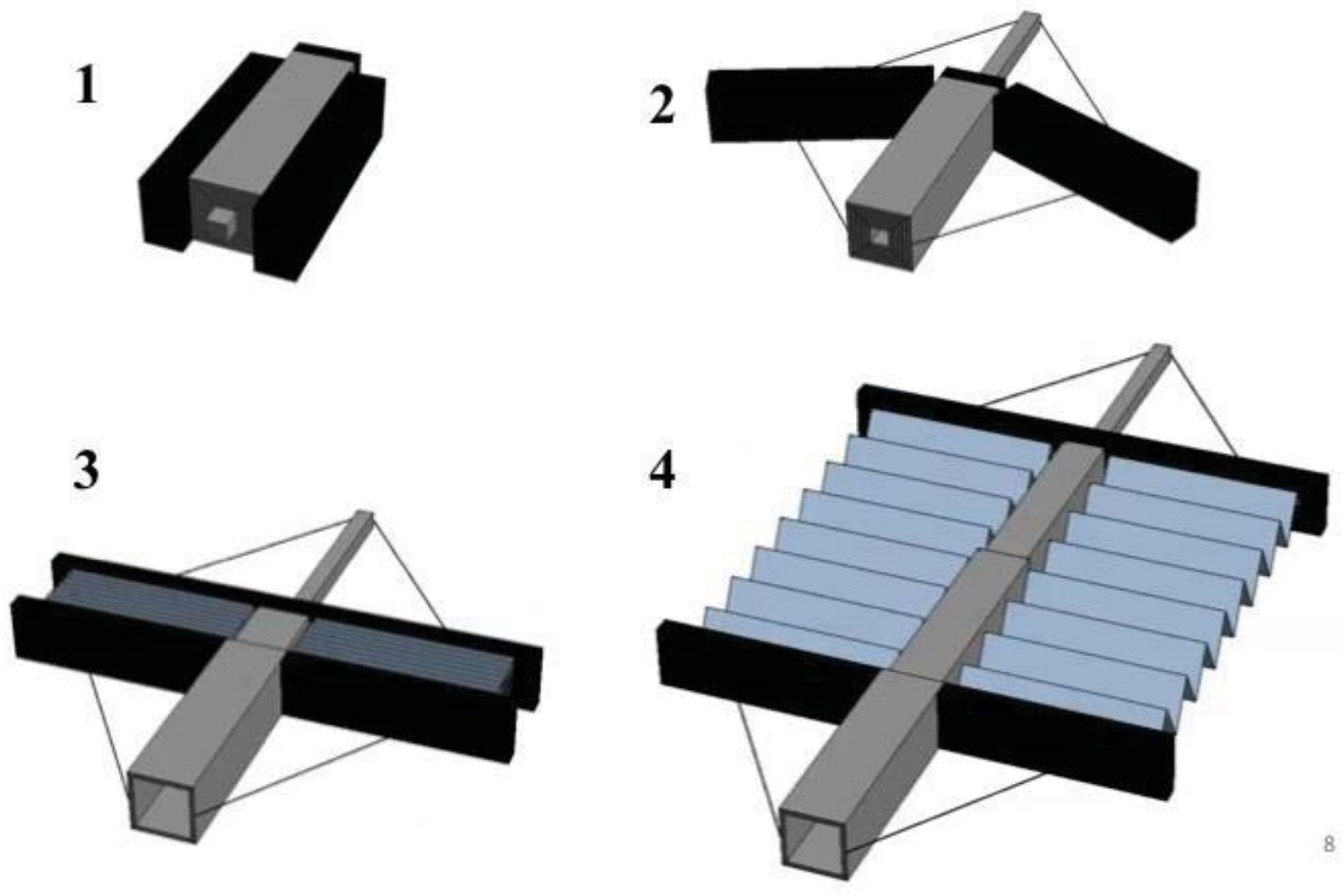

Figure 8. Compact telescoping array deployment sequence. 

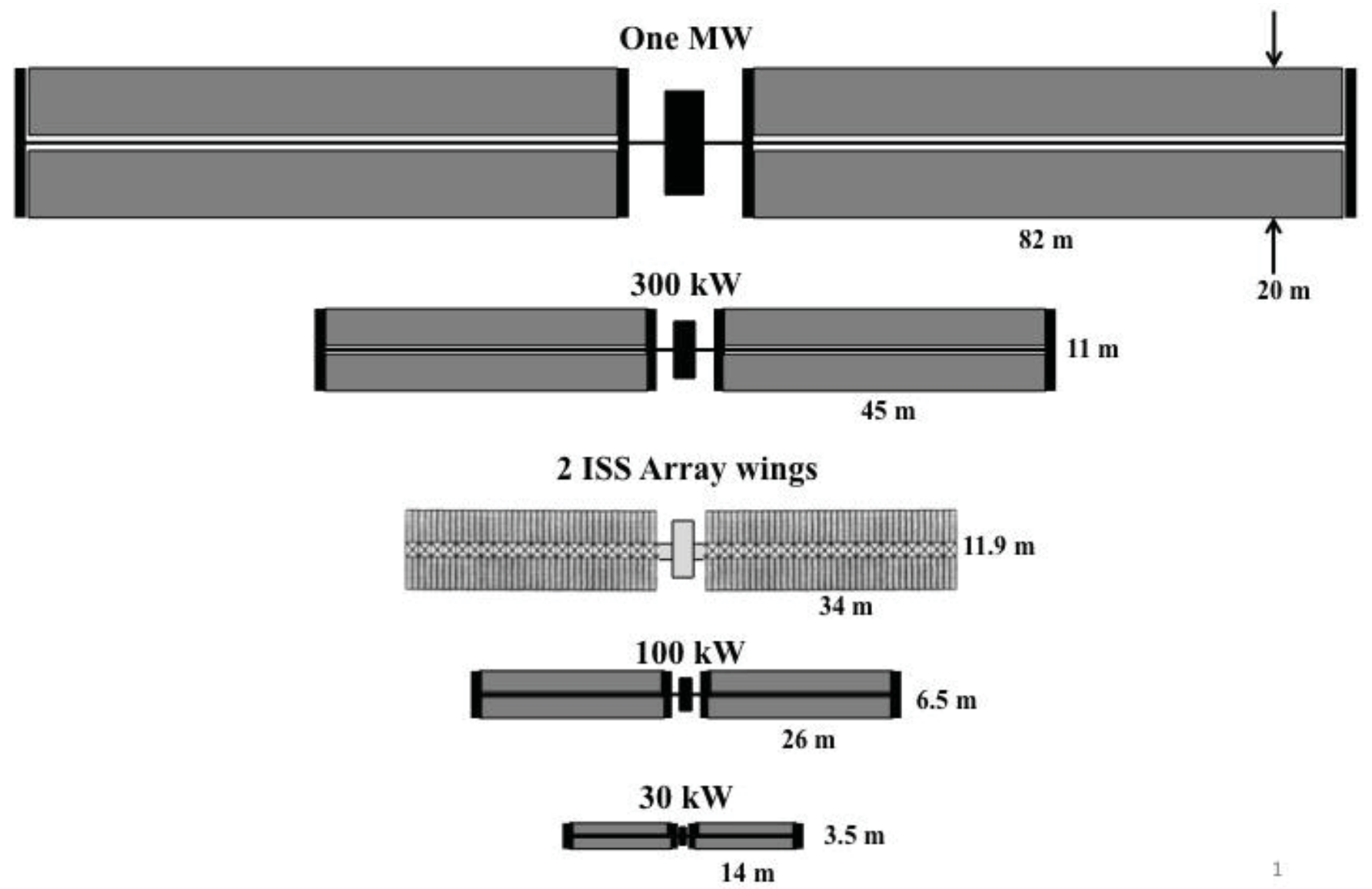

Figure 9. Solar array sizes versus power level.

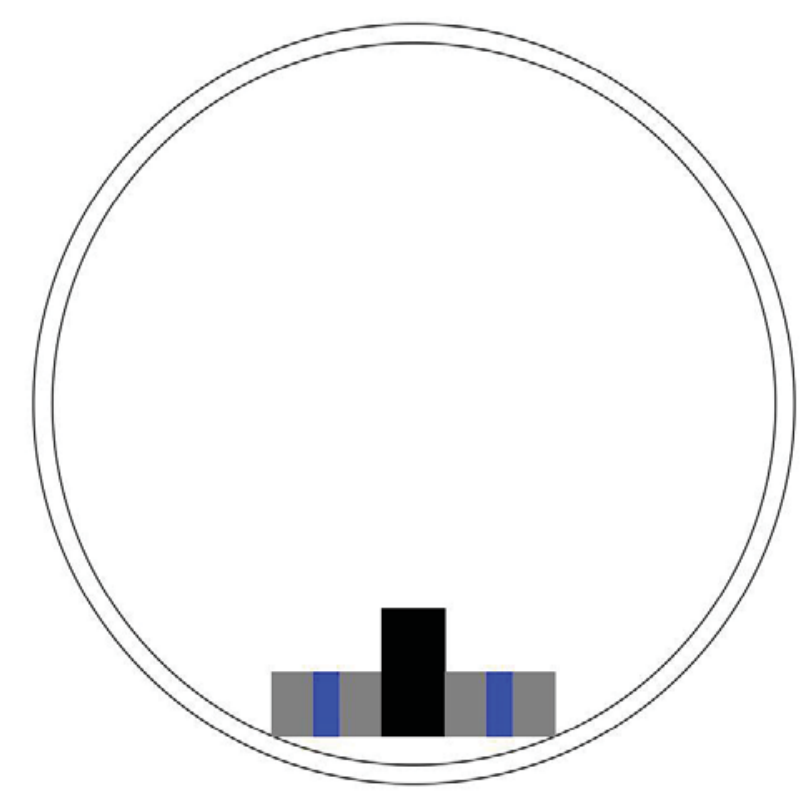

Figure 10. Cross-section of a $500 \mathrm{~kW}$ solar array wing shown inside of a 5-meter diameter launch shroud. The length of the packaged solar array wing is approximately 10 meters. 


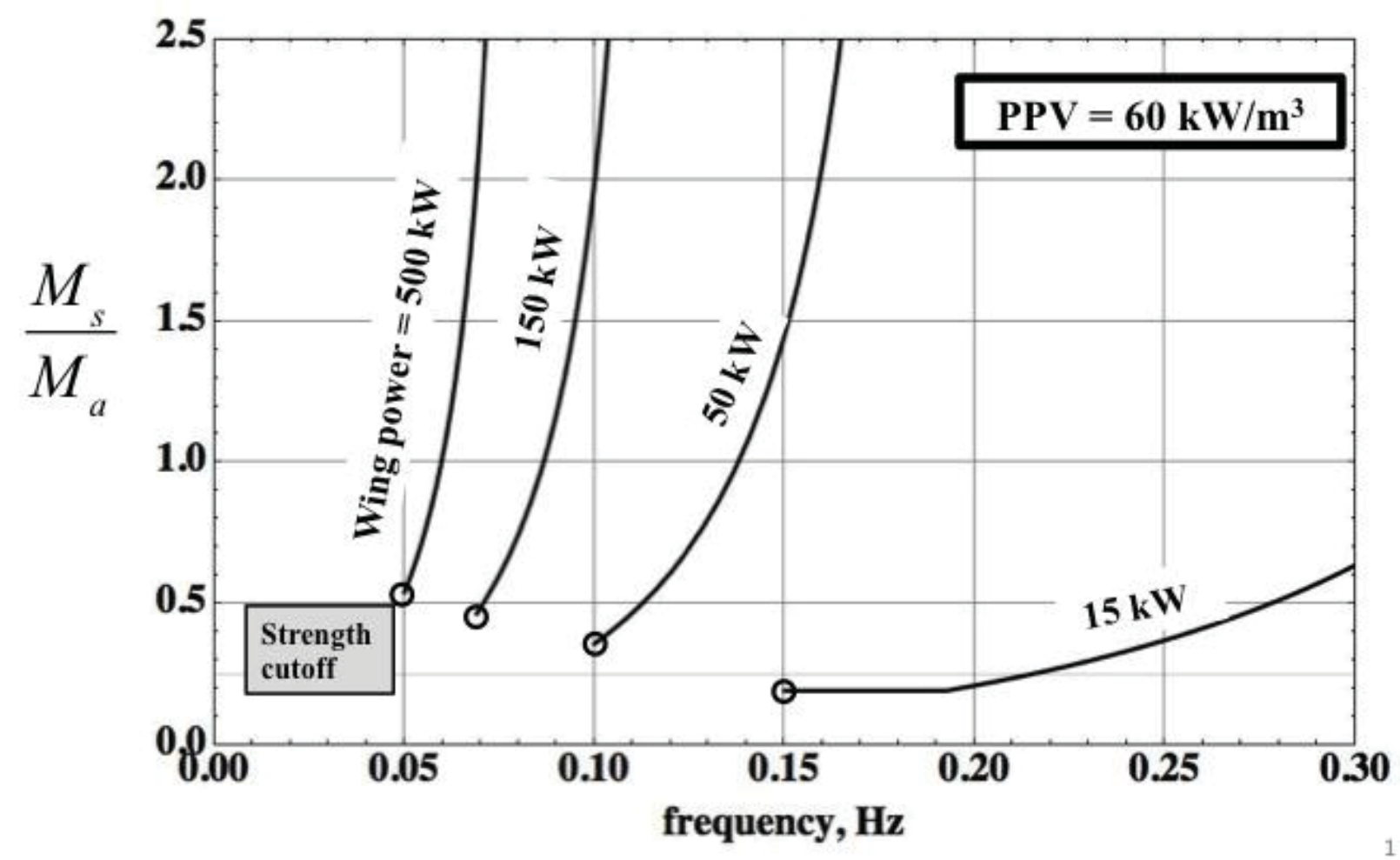

Figure 11. Compact telescoping array structural mass fraction (Mf) versus frequency.

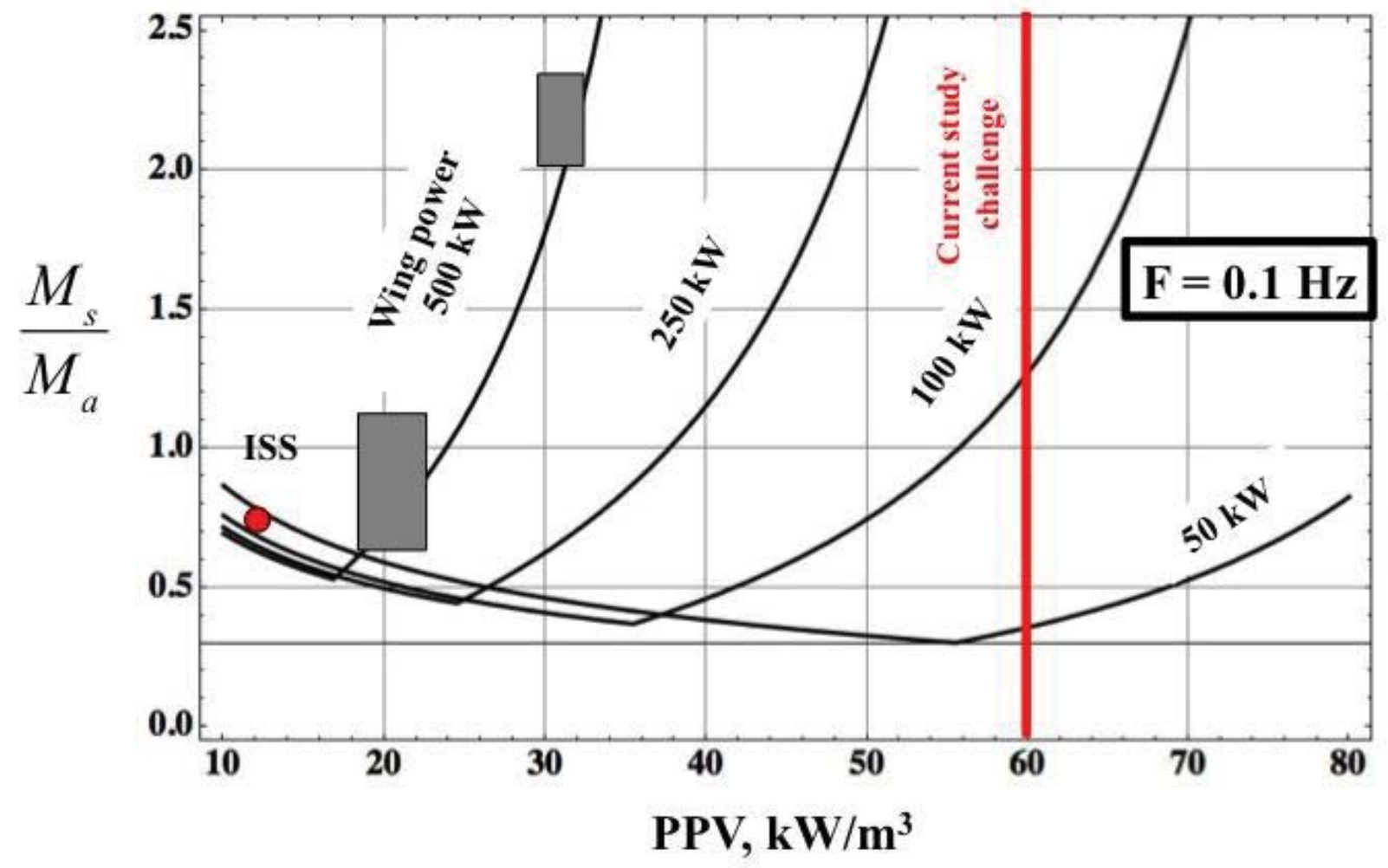

Figure 12. CTA structural mass fraction as a function of packaging volume for $f=0.1 \mathrm{~Hz}$. 


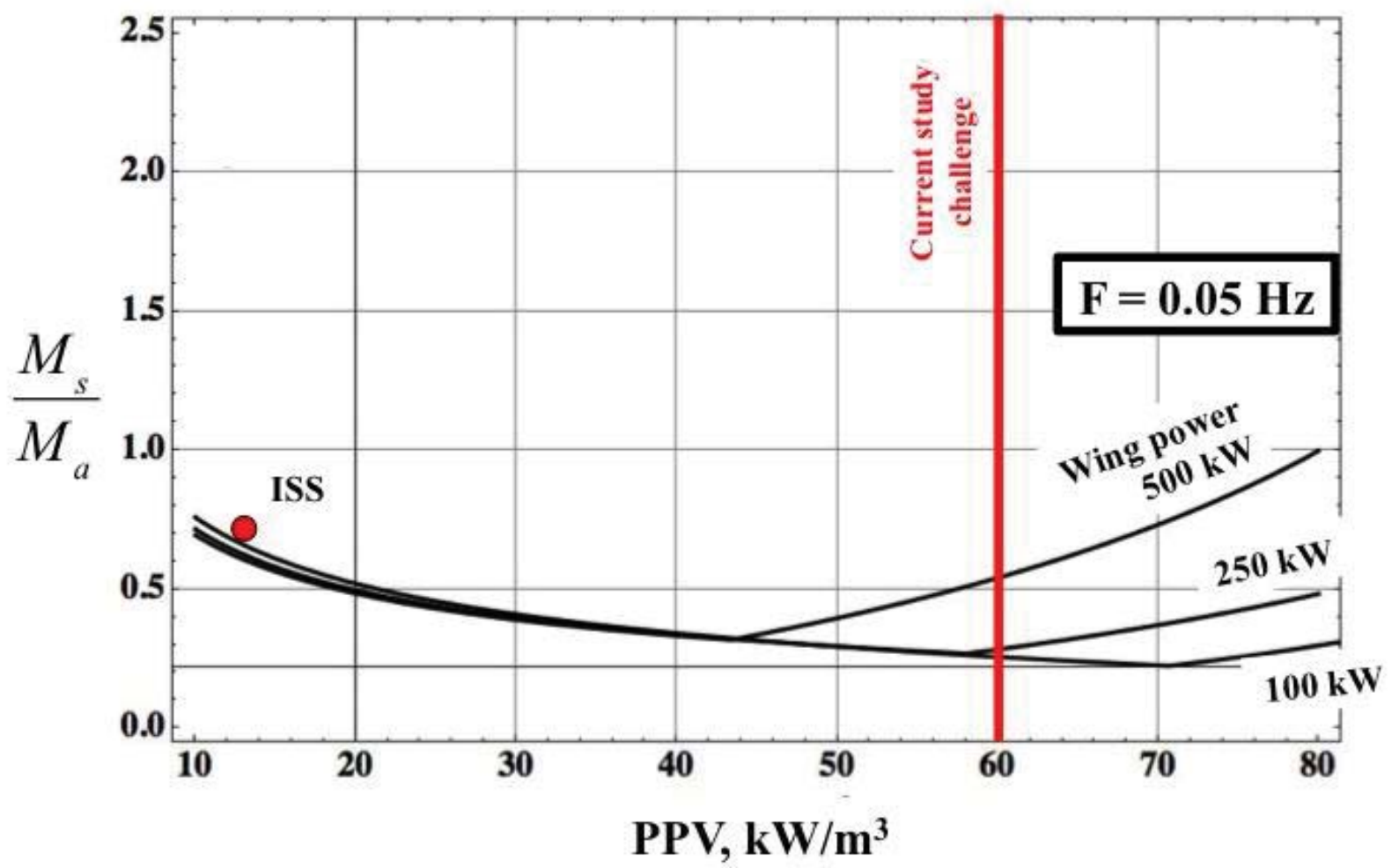

Figure 13. CTA structural mass fraction versus packaging volume for $f=0.05 \mathrm{~Hz}$.

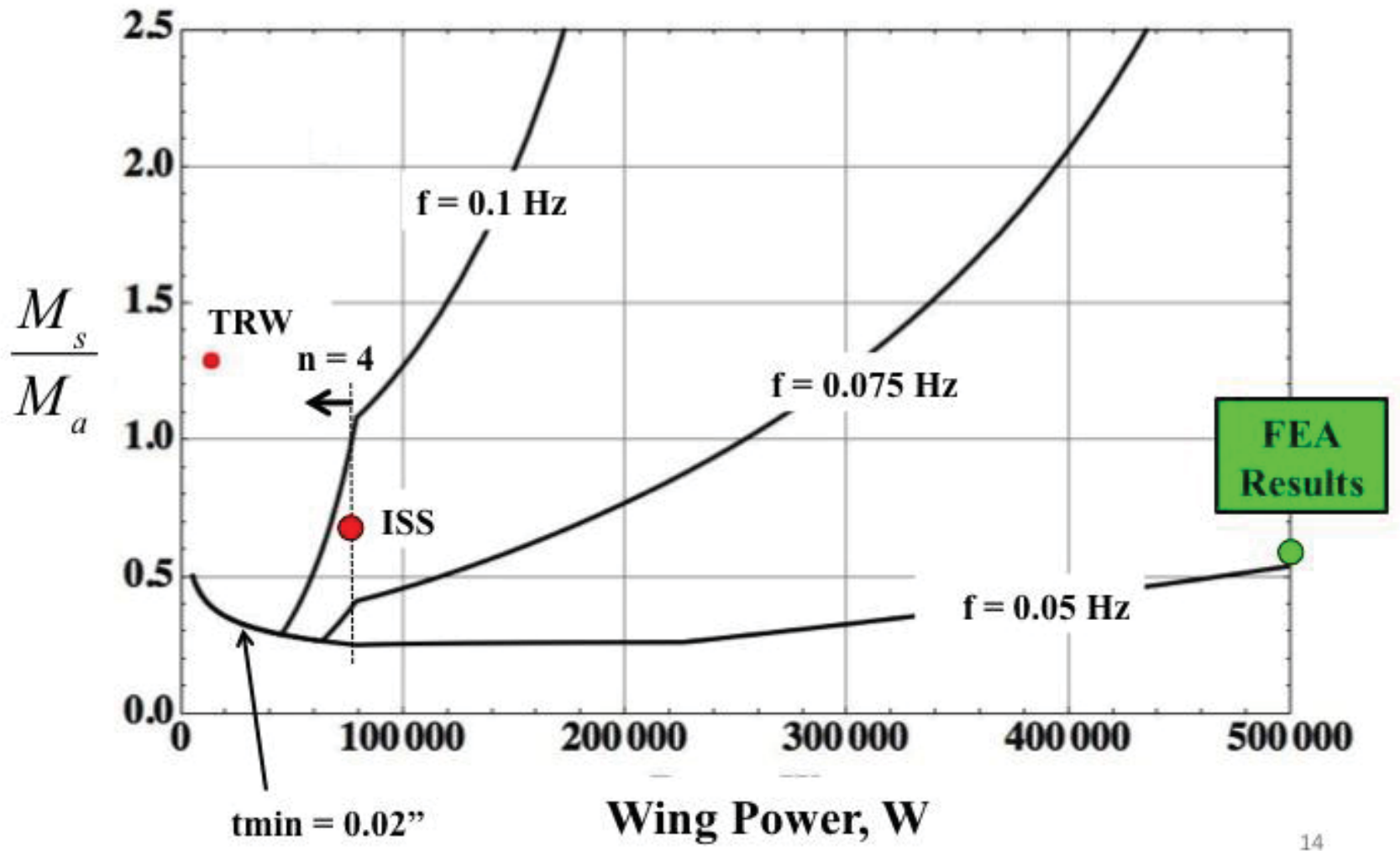

Figure 14. CTA structural mass fraction versus wing power for several frequency values. 


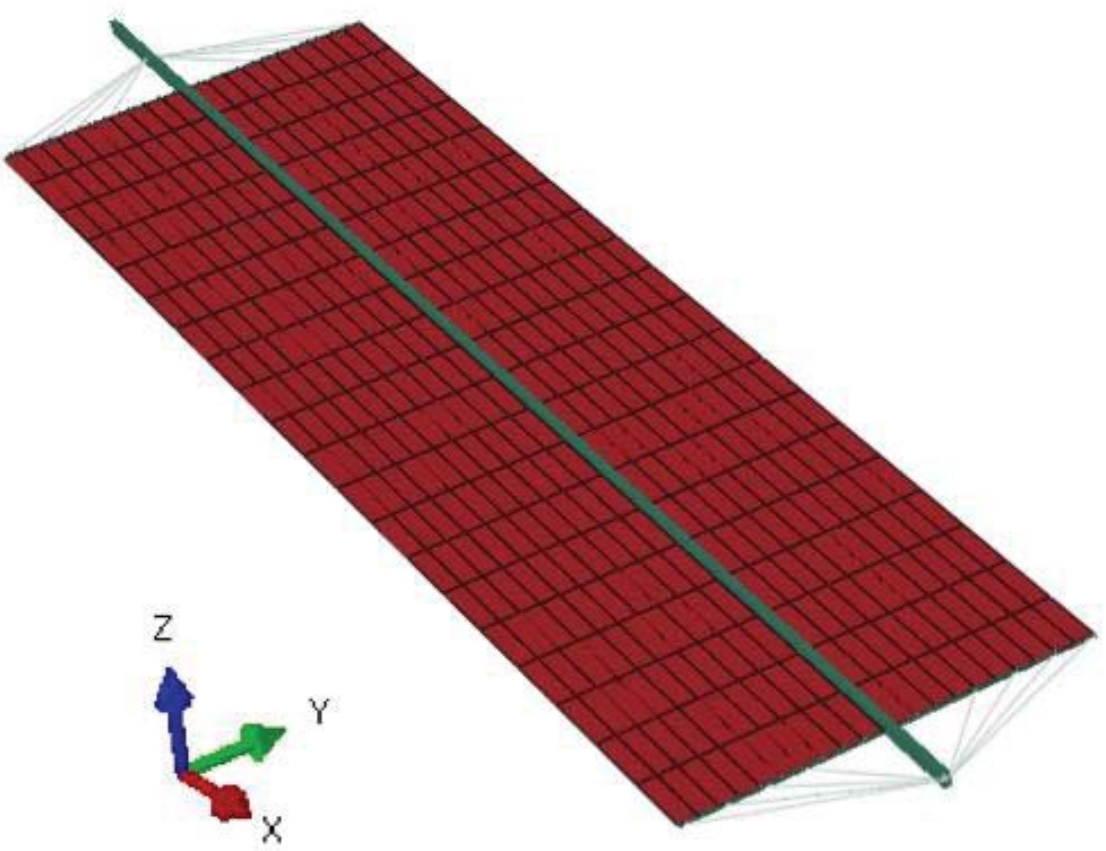

Figure 15. Finite element model used to verify simple analysis model used in design studies. 
Appendix A1 - Mathematica code for sizing the compact telescoping solar array

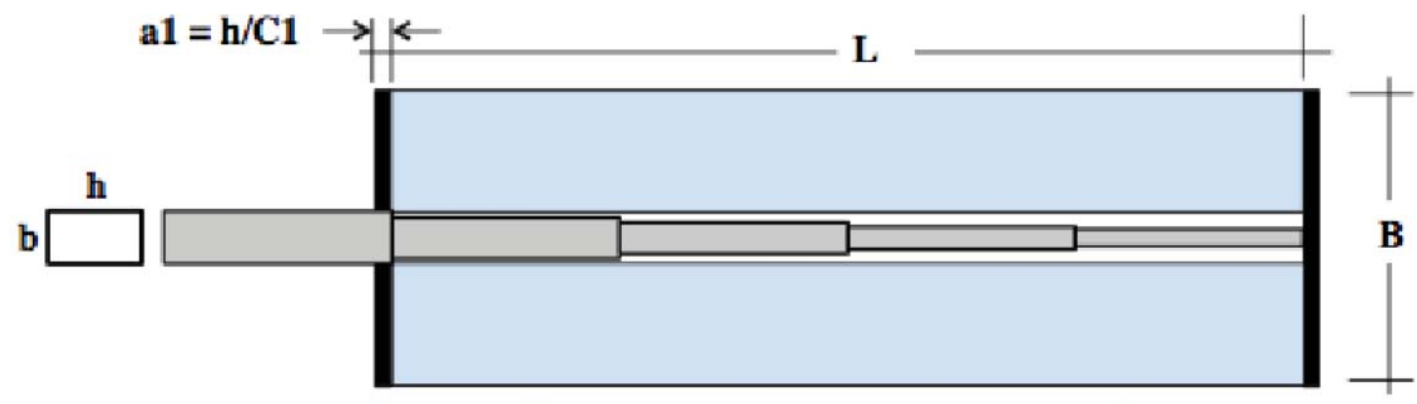

$n=$ number of telescoping section within array, $n=4$ for this example

Figure A-1. Overall dimensions of solar array.

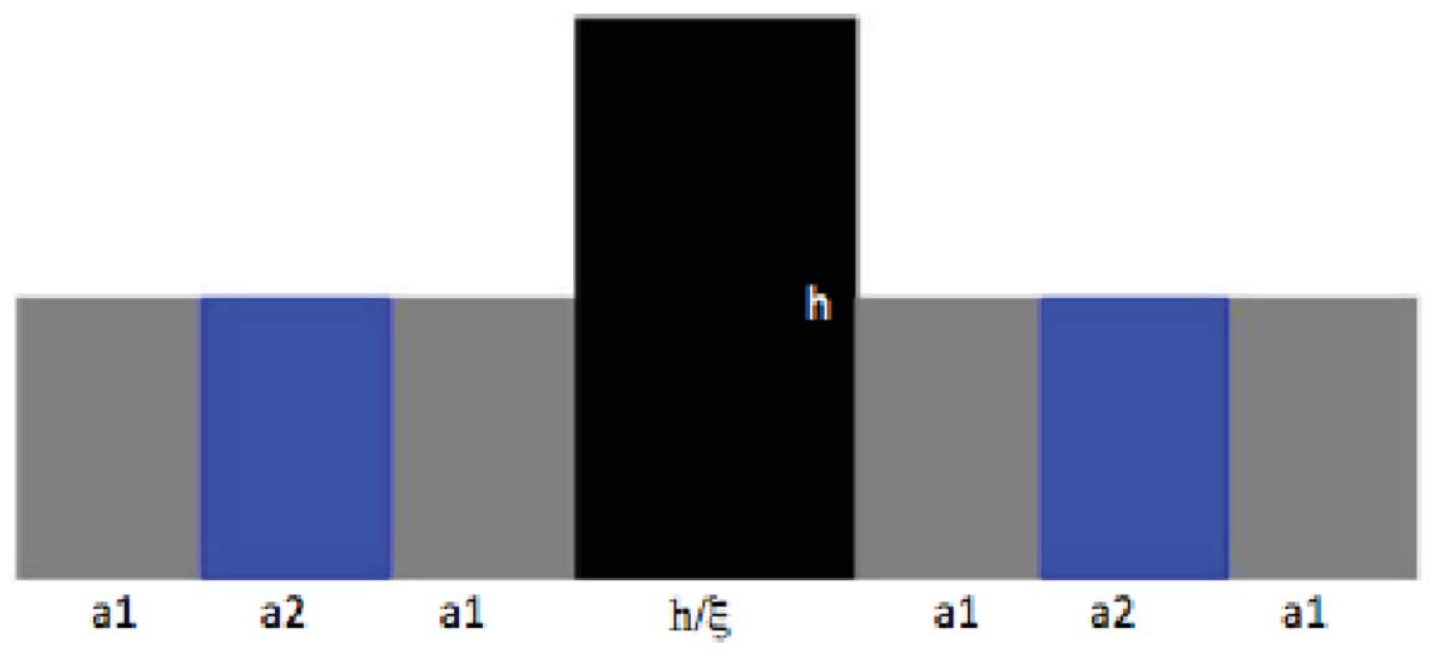

Figure A-2. End view of packaged array with major dimensions. 
Appendix A2 - Solve for array dimensions required to satisfy power and PPV constraints.

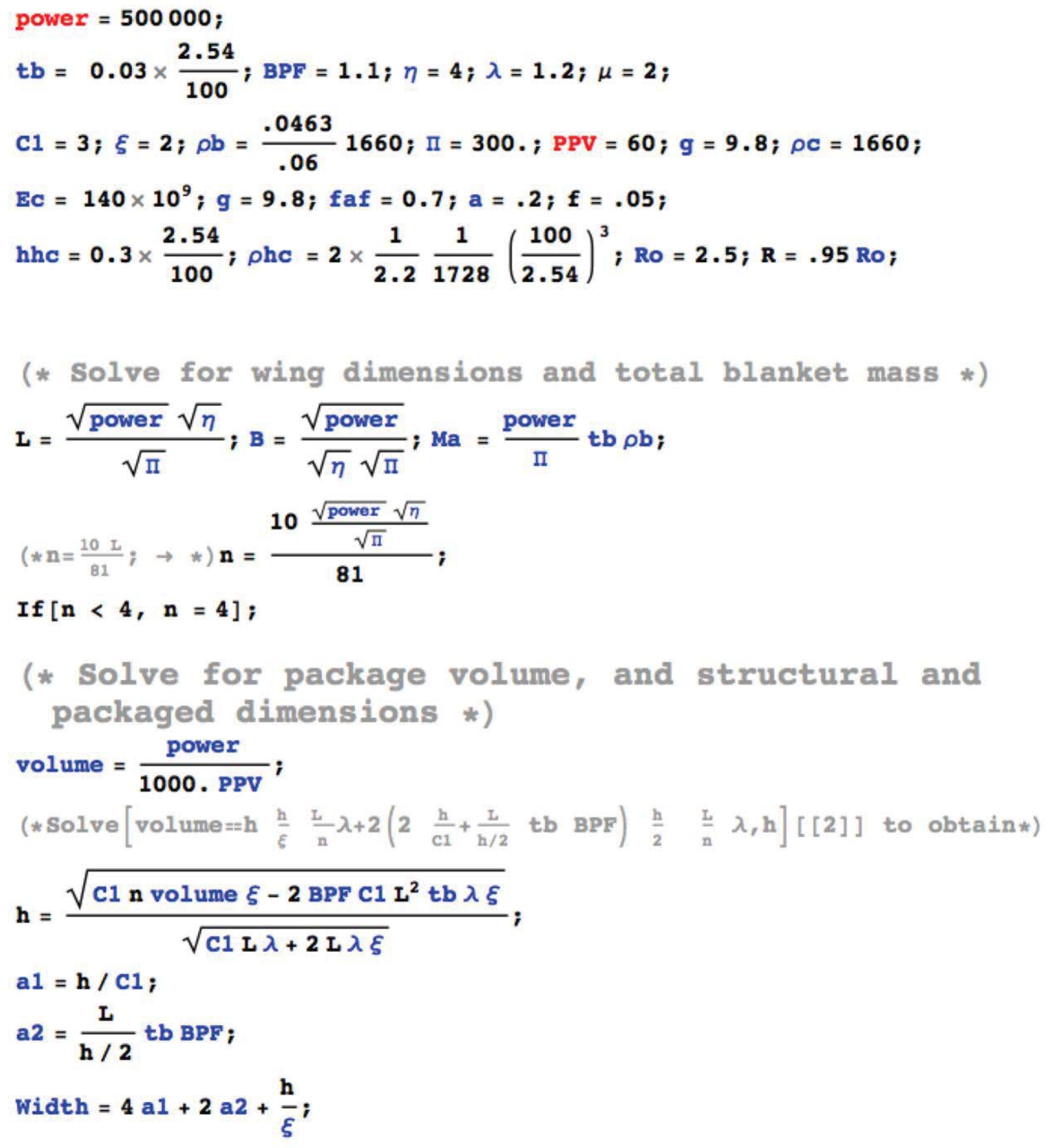


Appendix A3 - Solve for primary support structure wall thickness required to satisfy frequency constraint.

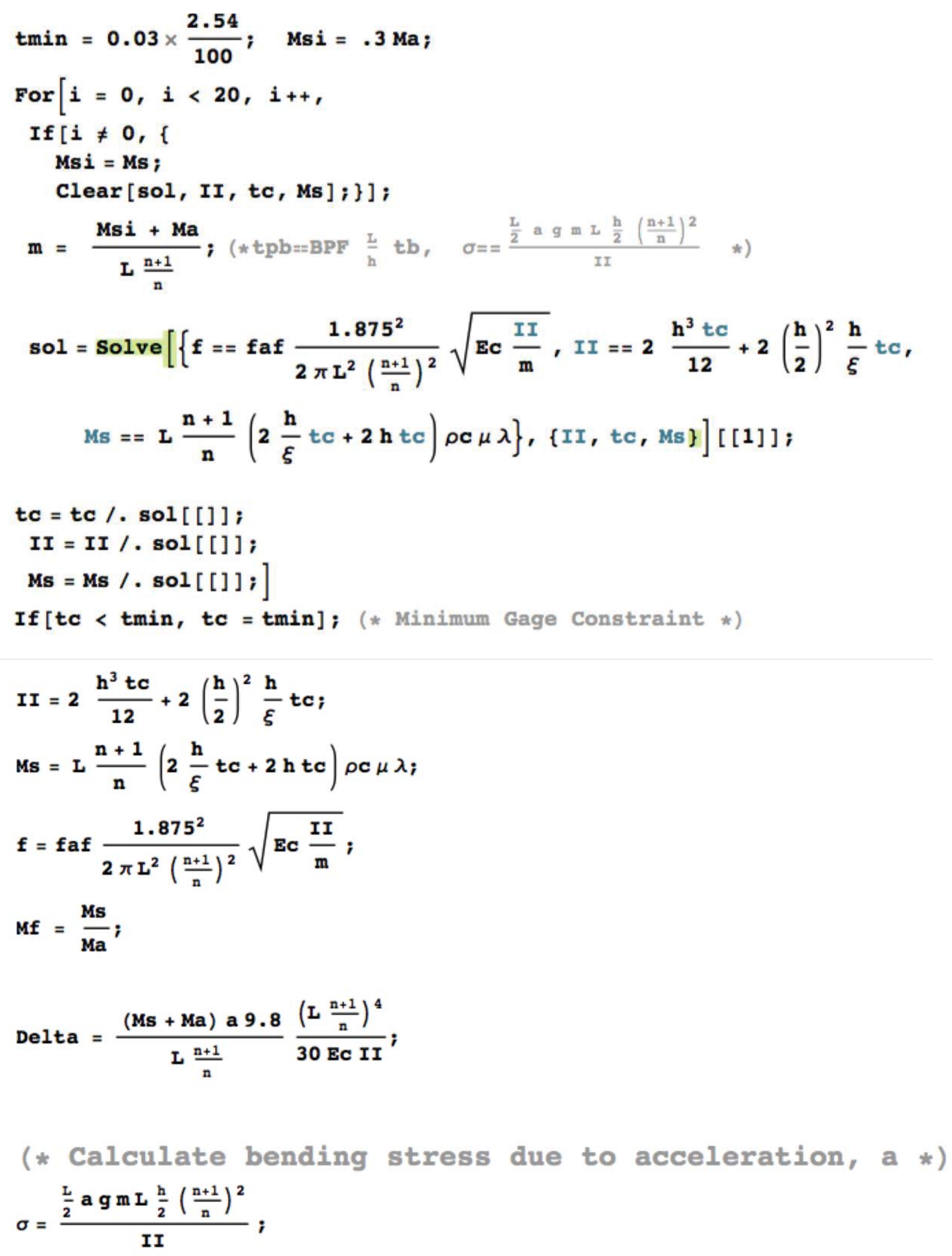




\section{Appendix A4 - Print statements.}

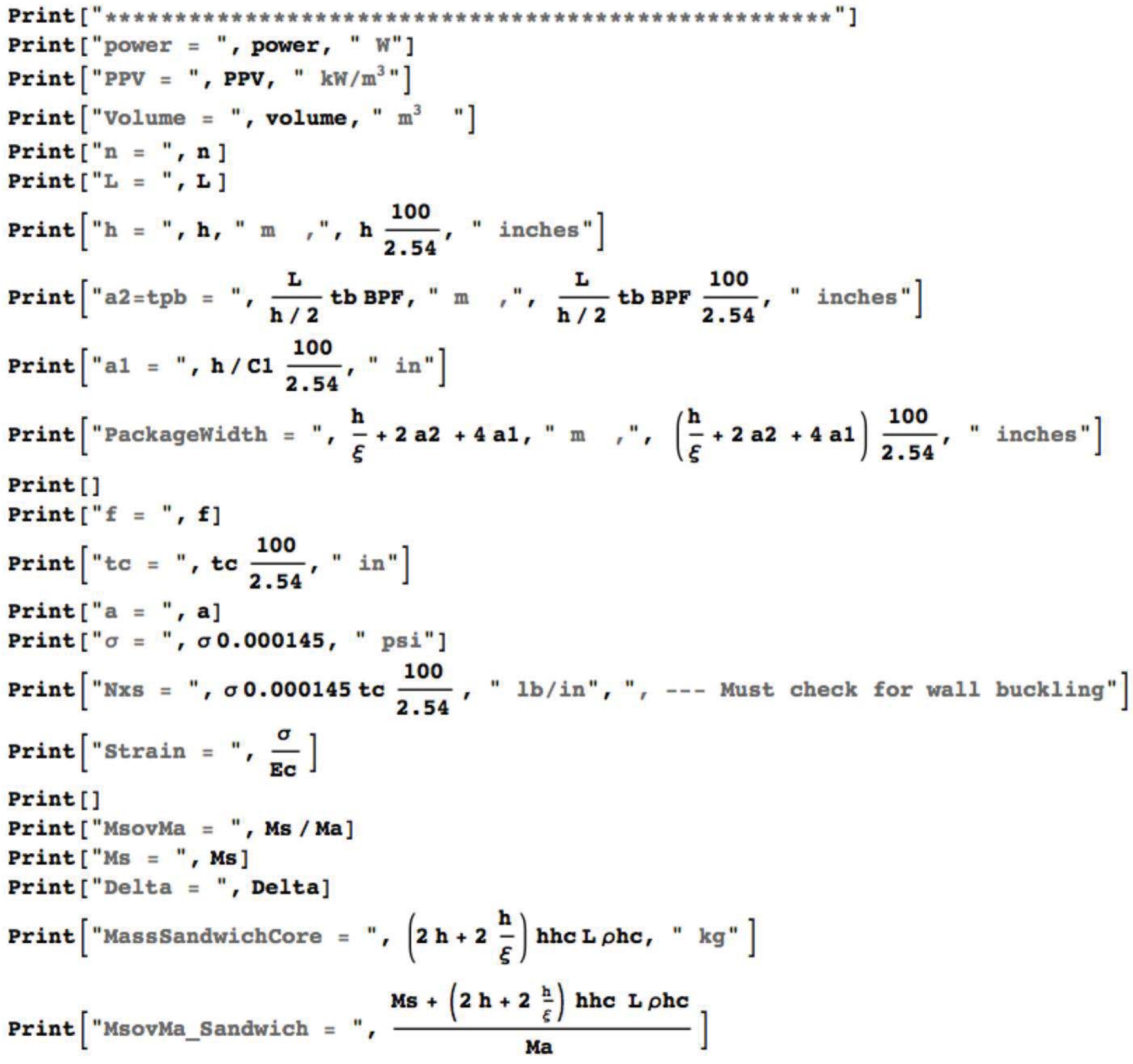




\section{Appendix A5 - 500 kW wing results}

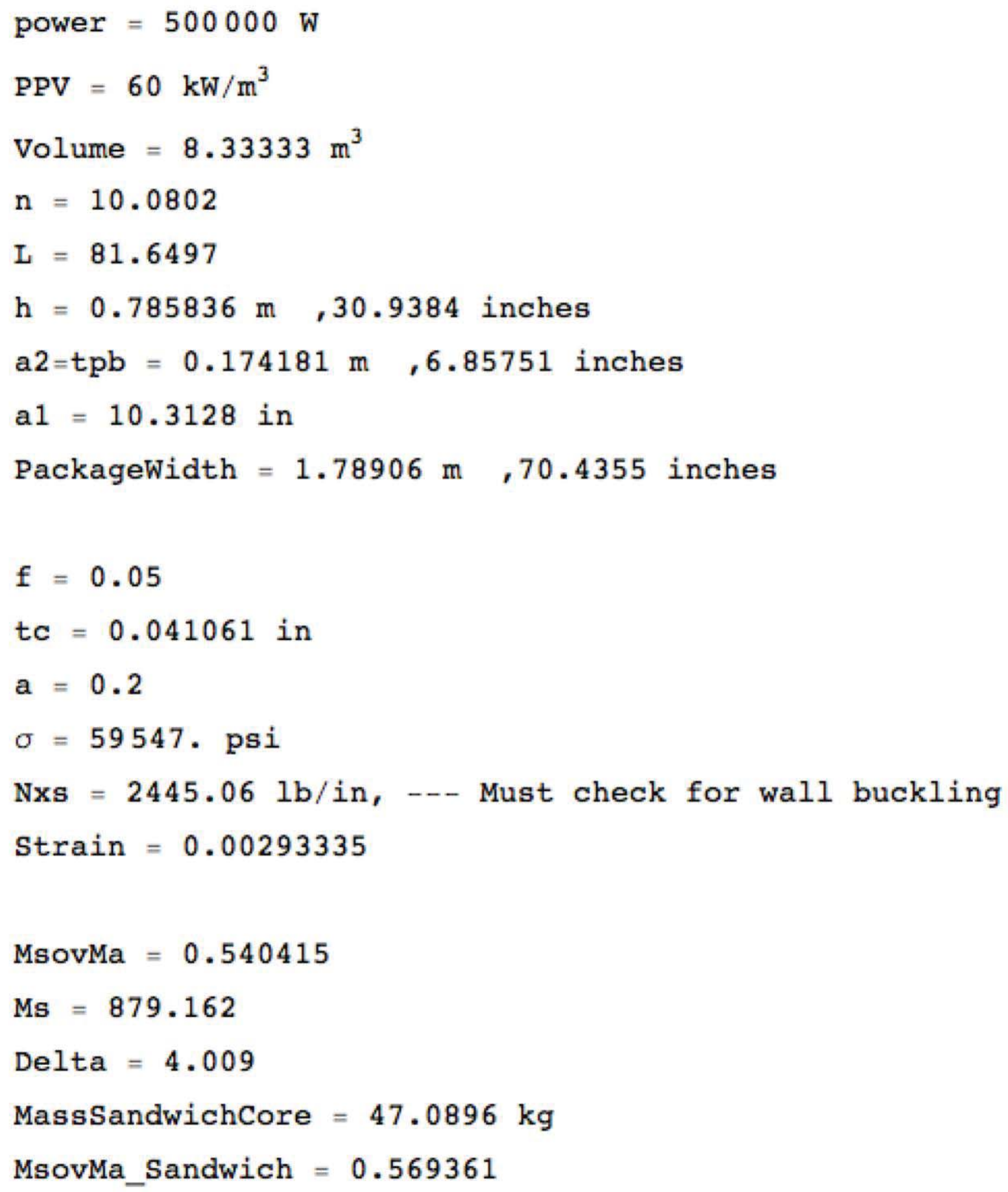

